Our recent experience in San Francisco has been that intensive management of these small infants (500-1000 $\mathrm{g}$ birth weight) results in survival of more than $60^{\circ}$ (see table). Infants born outside the regional perinatal centre

Neonatal mortality of infants of $500-1000 \mathrm{~g}$ birth weight admitted to intensive care unit, San Francisco Children's Hospital

\begin{tabular}{|c|c|c|c|c|c|}
\hline \multirow{2}{*}{ Year } & \multicolumn{2}{|c|}{ Inborn } & \multicolumn{2}{|c|}{ Outborn } & \multirow{2}{*}{$\begin{array}{c}\text { Total } \\
\text { Survival }\end{array}$} \\
\hline & No & Lived & No & Lived & \\
\hline $\begin{array}{l}1972 \\
1973 \\
1974 \\
1975 \\
1976 \\
1977 \text { (to } \\
\text { November) }\end{array}$ & $\begin{array}{r}15 \\
10 \\
6 \\
4 \\
13 \\
11\end{array}$ & $\begin{array}{l}2 \\
0 \\
1 \\
2 \\
7 \\
9\end{array}$ & $\begin{array}{r}12 \\
9 \\
9 \\
12 \\
20 \\
12\end{array}$ & $\begin{array}{r}3 \\
4 \\
1 \\
4 \\
12 \\
7\end{array}$ & 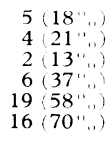 \\
\hline
\end{tabular}

("outborn") fare less well (during 1976November $197759^{\circ}$, survival compared with $67^{\circ}$ for those inborn). As regards morbidity, we have been able to follow up 13 of 17 inborn infants in this weight group for periods ranging from six months to six years and found 12 to appear normal. Of 29 outborn infants, 22 have been followed up to the same age and 14 of these were considered normal.

Our mortality figures up to 1975 are similar to those for England and Wales reported by Dr Gordon, but in 1976 and 1977 there appears to have been a marked improvement in outlook for these tiny infants. This we attribute to more thorough intensive care involving mechanical ventilation for considerable periods of time and careful use of intravenous feeding, though it is possible that other factors have played a part.

The problem is not that intensive care cannot improve survival for these small infants but rather that these facilities are not available to all mothers about to deliver prematurely at any time after 26 weeks of gestation. We are firmly convinced that all high-risk mothers should be delivered in regional centres where intensive care facilities are available. When it is not possible to use the uterus as the transport incubator, then early transfer of these immature babies to a regional newborn intensive care unit in well-equipped ambulances is the next best alternative.

T HiRATA

HenRy L HaLliday

Children's Hospital Medical Center

San Francisco, California

\section{Perinatal mortality rates by birth weight}

SIR,-Dr Ronald $\mathrm{R}$ Gordon has drawn attention recently (5 November, p 1202; 8 October, p 939; 21 May, p 1313) to the disproportionately greater fall in the number of "small" (less than $1000 \mathrm{~g}$ ) live births than total births which has occurred since 1962 in Sheffield and to a lesser extent in England and Wales.

Drs $\mathrm{R}$ Newcombe and I Chalmers (8 October, p 925) have shown, however, that in Cardiff the trend appears to be different from that in Sheffield. Using data on firstborn singleton infants born to primiparous mothers during the decade 1965-74, they have demonstrated a $12 \%$ increase in the proportion of infants weighing less than $2500 \mathrm{~g}$ during the second quinquennium. This increase in the proportion of low birth weight (LBW) babies in Cardiff would have tended to increase the perinatal morbidity, and a previous study by Dr Chalmers and his colleagues has shown that there has been little reduction in the perinata mortality rates there during the decade 1965-74.

The local experience in Wolverhampton appears to follow the Cardiff rather than the Sheffield trend when the total births among residents in the Wolverhampton Health Area are examined using the LHS27 returns made annually to the Department of Health and Social Security. The accompanying figure use the two-year moving means because the annual numbers are small and shows that, although the total number of births began to fall from 1968, the number of LBW babies did not begin to fall until 1972, whereas in Sheffield this fall began in 1962 well in advance of the fall in total births.
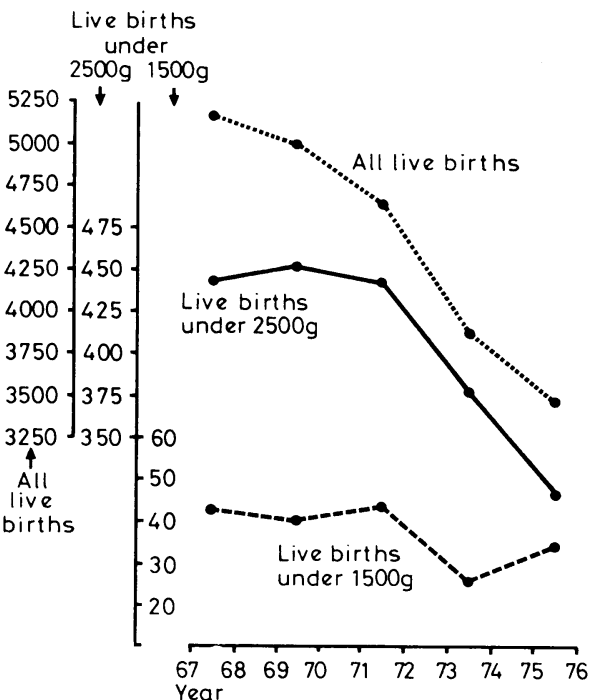

Live births to Wolverhampton residents (two-year moving averages).

Furthermore, perinatal deaths among Wolverhampton residents have been monitored through a local study since 1970 and it is clear that the perinatal mortality rates for babies of mothers born abroad and for babies of mother born in the UK do not differ greatly. For example, when births and perinatal deaths are aggregated for 1970-6 the stillbirth rates are respectively 19 and 17 per 1000 (all mothers 17 per 1000 ) and the perinatal (stillbirths and first-day deaths) rates are 27 and 24 per 1000 (all mothers : 24 per 1000). Using the data from the Office of Population Censuses and Surveys for the same period, the perinatal rate for all mothers is 30 per 1000 and it would seem reasonable to assume that within this population the $75 \%$ of babies from mothers born in the UK have a perinatal mortality rate of 30 and the $25 \%$ of babies from mothers born abroad (mainly India) have a rate of 33 per 1000 .

At a national level the decline in annual births during the 1970 s (a drop of $25 \%$ in six years) has been the result of a very substantial decrease in births in the lower (manual) socia classes and a smaller decline in the higher (non-manual) social classes.' As the proportion of socially disadvantaged mothers is greates in social classes IV and V it would be expected that both the proportion of LBW babies and the associated perinatal morbidity would be reduced during the period of falling births. It is paradoxical, however, that in some areas like Wolverhampton and Cardiff there has been little change during the last decade in either the proportion of LBW babies or the perinatal mortality rate.

The obvious conjecture is that there are factors operating to maintain the raised leve of LBW babies and the perinatal mortality associated with them, so that as the LBW babies attributable to socially disadvantaged mothers are reduced they appear to be replaced by babies whose mothers come from higher social classes. These factors, as yet unidentified, could be a reason why the proportion of LBW babies and the associated perinatal mortality have not fallen generally in England and Wales as much as, for example, in Sheffield.

F N GARRATT

E A JONES

Wolverhampton Area Health Authority Administrative Offices, New Cross Hospital,

Wolverhampton

Pearce, D, and Brittain, M, Population Trends, 1977, No 7.

\section{Still dissolving discs?}

SIR,-Mr E Shephard (26 November, p 1419) says that " $95 \%$ of our patients" recover with conservative treatment "at the proper time." Lucky man! Prolapsed intervertebral disc is ideally an operative diagnosis; otherwise the prolapse may be located by myelography, but the latter is usually reserved as a preliminary to operation. Therefore most patients treated conservatively have no objective evidence for the diagnosis, however suggestive the clinical signs may be. Can Mr Shephard say what happens to his patients after they recover? How many attacks do they have later, how long do they last, and how much working time is lost in total? What degree of disability qualifies as "recovery"? And how long is "the proper time"?

I think that no answers to these questions have been published. The painful disc with no nerve supply ${ }^{1}$ and disc prolapse remaining when the patient has recovered" are only two points which make this a difficult subject to analyse. Very little has been reported about the natural history of low back pain, and without that knowledge it seems difficult to argue that any method of treatment is superior or even effective.

E C Cawte

Ibstock, Leicester

1 Kumar, S, and Davis, P R, Fournal of Anatomy, 1973 ,
114,47 . ${ }^{2}$ Falconer, M A, McGeorge, M, and Begg, A C, Fournal of $N$,

\section{Ganglia}

SIR,-Y Your leading article (26 November, $p$ 1370) ignores a number of advances in the understanding of the mechanism and treatment of ganglia.

As long ago as 1840 Adams $^{1}$ suggested that popliteal cysts were connected to the joint by a valvular opening and in 1970 Jayson and Dixon ${ }^{2}$ demonstrated valvular connections allowing effusions to be pumped $f \mathrm{Jm}$ the knees into the cysts but not to return. They suggested that the valve might be of the Bunsen type, consisting of a narrow curved passage the walls of which collapse under direct cyst pressure. A similar pathogenesis for ganglia seemed likely, but this was not 